\title{
Scrotal hemorrhage after testicular sperm aspiration may be associated with phosphodiesterase-5 inhibitor administration: a retrospective study
}

Yong-tong Zhu' ${ }^{1 \dagger}$, Rui Hua ${ }^{1 \dagger}$, Song Quan ${ }^{1}$, Wan-long Tan ${ }^{3}$, Qing-jun Chu ${ }^{1 *}$ and Chun-yan Wang ${ }^{2^{*}}$

\begin{abstract}
Background: Scrotal hemorrhage after testicular sperm aspiration (TESA) is uncommon in clinical operation. Phosphodiesterase-5 inhibitors (PDE5i) are commonly given to men who have difficulty providing a sperm sample for assisted reproductive technique such as in vitro fertilization. In this study, we examine the incidence of scrotal hemorrhage after TESA in men who received a PDE5i.

Methods: In this retrospective study, 504 men with TESA operation in Center for Reproductive Medicine, Nanfang Hospital, Southern Medical University were collected. Men in the drug group had taken orally PDE5i before TESA. Men in the control group only operated TESA. The testis volume, coagulation function were measured. Sonographic examination with Doppler imaging was performed when scrotal hemorrhage appeared.

Results: A total of 504 men with a mean age of $28.63 \pm 4.22$ years were included in the analysis. Of these, 428 did not receive a PDE5i prior to TESA and 76 received a PDE5i prior to TESA. Measures of coagulation function were not different between the groups. The incidence of hemorrhage was $0.0 \%$ in the control group and the drug group was $5.3 \%$. The incidence of hemorrhage between two groups was different significantly $(P=0.000)$.

Conclusion: In summary, the results of this study suggest that a PDE5i administration increases the risk of scrotal hemorrhage in men undergoing TESA, although the study design does not allow drawing a conclusion of cause and effect. Given the potential risk of scrotal hemorrhage after the ingestion of PDE5i, it may be wise not to administer it to men in whom a TESA may be performed.
\end{abstract}

Keywords: Scrotal hemorrhage, Testicular sperm aspiration, Phosphodiesterase-5 inhibitor

\section{Background}

Over the past several decades, in vitro fertilization and intracytoplasmic sperm injection (ICSI) have come into routine practice. On the day of oocyte retrieval, the male partner is typically asked to provide sperm by masturbation. However, a small number of males are able to provide sperm by masturbation due to psychologic stress and

\footnotetext{
* Correspondence: 13763339658@163.com; zhuyongtong@sina.com ${ }^{\dagger}$ Equal contributors

${ }^{1}$ Reproductive Medicine Center, Department of Obstetrics and Gynecology, Nanfang Hospital/ The First School of Clinical Medicine, Southern Medical University, Guangzhou, China

${ }^{2}$ Department of Neurology, Integrated Hospital of Traditional Chinese

Medicine, Southern Medical University, Guangzhou, China

Full list of author information is available at the end of the article
}

anxiety. These individuals are generally provided psychological guidance and administered a phosphodiesterase- 5 inhibitor (PDE5i). When these measures are not successful, testicular sperm aspiration (TESA) is performed. Unfortunately, several males experienced scrotal hemorrhage after TESA.

As the worsening of the doctor-patient relationship in China, the violence in hospitals in China was growing and Chinese doctors were under tremendous stress [1]. It is necessary to look for the possible reason of scrotal hemorrhage after TESA and avoid such events. Surprisingly, none of azoospermia patients appeared scrotal hemorrhage after biopsy with the same TESA operation. It is assumed that scrotal hemorrhage after TESA may 
be associated with PDE5i administration. To our knowledge, reports about such cases are scarce. Therefore, this retrospective study was designed to summarize the data and answer this question.

\section{Methods}

\section{Study design}

We retrospectively reviewed the records of 504 men who underwent TESA at the Center for Reproductive Medicine, Nanfang Hospital, Guangzhou, China From 2012 to 2015. This study was approved by the Clinical Medical Local Ethical Review Committee of the Southern Medical University. Informed written consent was provided by all men in the study.

The men were classified into 2 groups; those who received a PDE5i before TESA and those who did not receive a PDE5i. Briefly, if men were unable to provide a sperm sample by masturbation due to stress/anxiety, and met the criteria described below, they were given tadalafil $20 \mathrm{mg}$ and attempted to provide a sample by masturbation again. If a sample could not be provided TESA was performed. The time from tadalafil administration to TESA was approximately $3 \mathrm{~h}$. The criteria of men in whom TESA was performed directly (not given the option of masturbation, control group) are described below.

Five hundred four men in this study were all infertility more than 1 year. Inclusion criteria for the drug group were: (1) had ejaculation by masturbation successfully more than twice, (2) were not able to ejaculation on the day of oocyte retrieval, (3) sperm were identified on semen analyses. Inclusion criteria for the control group were: (1) previously identified azoospermia, which contained obstruction azoospermia and non- obstruction azoospermia, or cryptozoospermia [2], (2) testis volumes were more than $8 \mathrm{~mL}$. Patients in the drug group were all sperm retrieval performed by TESA successfully. In order to compare consistently, patients with prior epididymal sperm aspiration (PESA), testicular sperm extraction (TESE) or micro-TESE were excluded from this study.

\section{TESA procedure}

The testis was anesthetized using 1\% lidocaine. A 23 gauge needle was passed through the scrotal skin into the testicular tissue. Suction was applied with a $5 \mathrm{~mL}$ syringe, and the backpressure was maintained by hand. The needle was pushed in different directions into the testicular tissue to obtain a sample. Once an adequate sample was obtained, the needle was slowly removed from the testis while the negative pressure was maintained (Fig. 1). The sample was placed on a sterile plate, and the small tubules recovered were picked up by an assistant using 2 pairs of fine tweezers. Sperm in

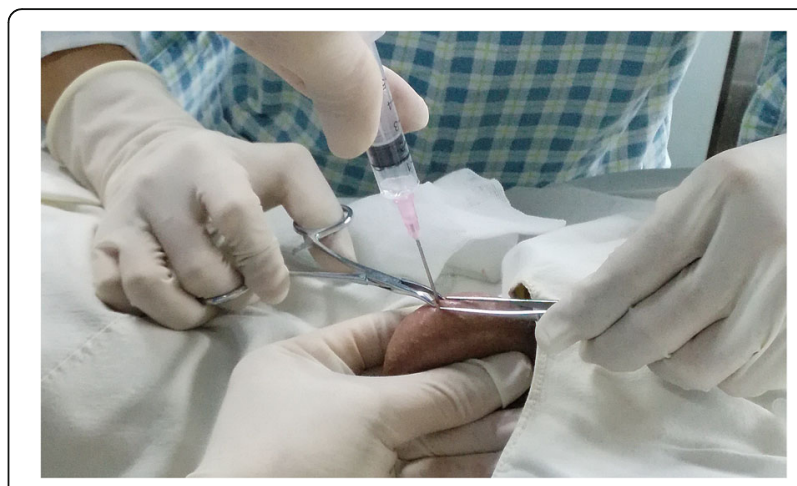

Fig. 1 The testicular sperm aspiration procedure

aspirated tissue was retrieved for ICSI or biopsy. All procedures were performed by Dr. Chu and Dr. Zhu.

\section{Follow-up}

Patients were given wound care instructions, and instructed to keep the puncture site dry and clean for 3 days. They were asked about light level of activity following the procedures, and abstained from strenuous exercise for 1 month. Patients were seen for follow-up at 3 day, 1 week, and 1 month after TESA. At the follow-up visits, examination of the scrotum was performed for identification of hematoma formation. Patients were also asked the following 2 questions at each visit: (1) Did you avoid the strenuous exercise? (2) Did you feel uncomfortable in your scrotum?

Sonographic examination with Doppler imaging was performed if physical examination was consistent with a scrotal hemorrhage. If a hemorrhage did not selfabsorb during 4- week period, an evacuation procedure was performed to remove the remaining blood products and clots.

\section{Statistical analysis}

Calculations were analyzed by using SPSS 19.0 software (SPSS Inc., Chicago, Illinois, USA). All numeric data were presented as the mean value \pm standard deviation. Frequencies were expressed as percentages. Students ttest was used for comparisons between 2 groups, and Fischer's exact test was used for comparison of proportions. Values of $P<0.05$ were considered to indicate statistically significant differences.

\section{Results}

A total of 504 men who received TESA from 2012 to 2015 were included in the analysis. Mean age of the men was $28.63 \pm 4.22$ years. The testis size, character and the presence of testicular mass, or asymmetry was assessed via manual palpation and orchidometer. Mean testis volume was $11.8 \pm 2.6 \mathrm{ml}$ (Table 1). Coagulation function, which contained Thrombin time, Activated partial 
Table 1 Characteristics of patients

\begin{tabular}{|c|c|c|c|c|}
\hline & Control group & Drug group & Overall & $P$ value \\
\hline Number & 428 & 76 & 504 & \\
\hline Age (years) & $28.40 \pm 3.86$ & $29.07 \pm 4.11$ & $28.63 \pm 4.22$ & $P>0.05$ \\
\hline Testis volume (mL) & $12.0 \pm 4.3$ & $11.5 \pm 2.9$ & $11.8 \pm 3.6$ & $P>0.05$ \\
\hline \multicolumn{5}{|l|}{ Coagulation function } \\
\hline Thrombin time (sec) & $15.24 \pm 2.11$ & $14.98 \pm 2.03$ & $15.11 \pm 2.14$ & $P>0.05$ \\
\hline Activated partial thromboplastin time (sec) & $25.71 \pm 3.23$ & $26.01 \pm 3.17$ & $25.89 \pm 3.26$ & $P>0.05$ \\
\hline International normalized ratio & $0.89 \pm 0.23$ & $0.91 \pm 0.22$ & $0.90 \pm 0.22$ & $P>0.05$ \\
\hline Prothrombin time (sec) & $11.65 \pm 1.09$ & $12.02 \pm 1.16$ & $11.87 \pm 1.18$ & $P>0.05$ \\
\hline Fibrinogen (g/L) & $2.55 \pm 1.03$ & $2.64 \pm 1.06$ & $2.62 \pm 1.07$ & $P>0.05$ \\
\hline Incidence of hemorrhage (\%) & $0(0.0)$ & $4(5.3)$ & $4(0.8)$ & $P=0.000$ \\
\hline
\end{tabular}

thromboplastin time, International normalized ratio, Prothrombin time and Fibrinogen were not different between the 2 groups. There were 428 men in the control group (no PDE5i) and 76 men in the drug group. The overall incidence of scrotal hemorrhage in the 504 men was $0.8 \%$. However, the incidence in the control group was $0.0 \%$, and in the PDE5i group was $5.3 \%$ ( 4 men $)(P=0.000)$. All 4 patients required an evacuation procedure. No other post-operative complications were noted.

\section{Discussion}

TESA, which was developed in 1992, is a method for retrieving sperm for use in assisted reproductive technology [3]. The procedure is also used to perform biopsy of the testis. Compared to TESE, TESA is a simpler procedure with minimal physiological consequences [4]. The majority of patients in the control group were obstructive patients. While azoospermic patients who had smaller testicular volume $(<8 \mathrm{~mL})$, especially in the setting of testicular hypofunction, TESE or micro-TESE would be more appropriate in these patients.

It has been reported that intra-testicular hematoma formation occurs in 29\% of diagnostic testicular biopsies [5]. However, scrotal hemorrhage was a relatively rare clinical event after TESA. In the current study of 504 TESA procedures, the incidence was only $0.8 \%$. The difference in rates may be due to the increased use of sonographic examination. Most patients do not feel uncomfortable after TESA, and routine sonographic examination is not performed, and thus small areas of hemorrhage maybe overlooked.

At our institution, during the period from 2013 to 2015, 76 men successfully ejaculated by masturbation more than 2 times, but they were not able to ejaculation on the day of oocyte retrieval. Patients on intracavernosal injection treatment had high withdrawal rates. The most common reason for withdrawal was poor response to the therapy, followed by the inconvenience of use [6].
So they did not receive such therapy in our centre. These men passed through a procedure of relaxation, given pornographic material, mood adjusting and PDE5i drug taking, selected TESA operation finally to retrieve sperm. Although the proportion of these men who developed a scrotal hemorrhage was only $2.6 \%$, no scrotal hemorrhage occured after same operation in the other 428 patients who did not receive a PDE5i. The results suggest that the scrotal hemorrhage in these 4 patients was related to the use of a PDE5i.

PDE5i, such as sildenafil (Viagra), vardenafil (Levitra) and tadalafil (Cialis), are used to treat erectile dysfunction. PDE5i increases nitrous oxide (NO) and cyclic guanosine monophosphate (cGMP) in the smooth muscles of the corpus cavernosum. For a PDE5i to be effective, sufficient sexual stimulation is essential [7]. The men who received a PDE5i still could not relax enough to achieve sexual arousal and could not successfully ejaculate. PDE5i are generally safe and well tolerated [8], have not been reported in association with scrotal hemorrhage events. As men were not able to ask to stop PDE5i before any surgical procedure, the rationale for excess bleeding in men taking PDE5i may not exist. Although our results showed a correlation between taking a PDE5i and scrotal hemorrhage, a cause-effect relationship could not be determined from the study design. However, the mechanism by which a PDE5i increases the risk of a scrotal hemorrhage may be as follows. First, a PDE5i results in vasodilation, and redistribution of arterial blood flow that is associated with rupture of vessels. Second, the NO and cGMP pathway might be responsible for inhibition of platelet aggregation and activation. Finally, PDE5i are considered as an antithrombotic agent [9].

The limitations of this study include its retrospective design. A prospective study should be done to validate our results. Although a large number of patients participated in this study, it appears as there were only 4 events (hematoma) in the 504 patients, which may be not enough to draw the conclusion and the results maybe only 
anecdotal. As ultrasound was only performed after the physician suspected a hematoma on physical exam post procedure. This may introduce significant bias and lack of certainty if there actually were many other patients that did not develop hematomas that were not detected by the clinician performing a 3 days post procedure exam. So it would be better to perform ultrasound exam at 3 days after TESA.

Although there were no sufficient evidences which could support that scrotal hemorrhage after TESA was caused by the administration of a PDE5i. Given the potential risk of scrotal hemorrhage after the ingestion of a PDE5i, it should be cautious to prescribe this medicine when the patient is likely to perform TESA. Patients were on anti-coagulants, anti-platelets, taking NSAIDs and ice scrotum may be beneficial after TESA. Although it is difficult to predict ahead who have difficulty producing a semen sample of the day of assisted reproductive technique, as all patients in the drug group had been able to produce ejaculated sperm twice prior to the procedure date. We should gain information by detailed inquiry. It would be better to prepare frozen sperm before the day of the retrieval once patient were categorized as masturbation difficulty males.

\section{Conclusion}

In summary, the results of this study suggest that a PDE5i administration increases the risk of scrotal hemorrhage in men undergoing TESA, although the study design does not allow drawing a conclusion of cause and effect. Given the potential risk of scrotal hemorrhage after the ingestion of PDE5i, it may be wise not to administer it to men in whom a TESA may be performed.

\section{Abbreviations}

CGMP: Cyclic guanosine monophosphate; ICSI: Intracytoplasmic sperm injection; NO: nitrous oxide; PDE5i: Phosphodiesterase type5 inhibitor; PESA: Epididymal sperm aspiration; TESA: Testicular sperm aspiration; TESE: Testicular sperm extraction

\section{Acknowledgments}

This research was supported by The Chinese Medical Association of Clinical Medicine Research Special Fund (No. 16020530669, 16020170633), Natural Science Foundation of Guangdong Province (No. 2016A030310403, 2015A030310367), Medical Scientific Research Foundation of Guangdong Province (No. A2016092), and Merck Serono China Research Fund.

\section{Availability of data and materials}

The dataset supporting the conclusions of this article was included within the article. Table 1 represented the data from which the conclusions were made. The datasets used and analysed during the current study available from the corresponding author on reasonable request.

\section{Authors' contributions}

$\mathrm{YZ}$ and $\mathrm{RH}$ contributed equally to this work. $\mathrm{YZ}, \mathrm{CW}, \mathrm{RH}, \mathrm{WT}$ and $\mathrm{QC}$ conceived and designed the study; $Y Z$ and $C W$ collected data; $Y Z, C W, R H$, WT and QC performed data analysis; YZ, CW, RH, SQ, WT and QC prepared the manuscript; All authors read and approved the final manuscript.

\section{Ethics approval and consent to participate}

This study was approved by the Clinical Medical Local Ethical Review Committee of the Southern Medical University. Informed written consent was provided by all men in the study.

\section{Consent for publication}

Consent to use the image in this publication was obtained from the patient.

\section{Competing interests}

The authors declare that they have no competing interests.

\section{Publisher's Note}

Springer Nature remains neutral with regard to jurisdictional claims in published maps and institutional affiliations.

\section{Author details}

${ }^{1}$ Reproductive Medicine Center, Department of Obstetrics and Gynecology, Nanfang Hospital/ The First School of Clinical Medicine, Southern Medical University, Guangzhou, China. ${ }^{2}$ Department of Neurology, Integrated Hospital of Traditional Chinese Medicine, Southern Medical University, Guangzhou, China. ${ }^{3}$ Department of Urology, Nanfang Hospital, Southern Medical University, Guangzhou, China.

Received: 14 June 2016 Accepted: 17 January 2018

Published online: 06 February 2018

\section{References}

1. Yang T, Zhang H, Shen F, Li JW, Wu MC. Appeal from Chinese doctors to end violence. Lancet. 2013;382(9906):1703-4.

2. Zhu YT, Luo C, Li Y, Li H, Quan S, Deng YJ, Yang Y, Hu YH, Tan WL, Chu QJ. Differences and similarities between extremely severe oligozoospermia and cryptozoospermia in intracytoplasmic sperm injection. Asian J Androl. 2016; 18(6):904-7.

3. Nowroozi MR, Ahmadi H, Ayati M, Jamshidian H, Sirous A. Testicular fineneedle aspiration versus testicular open biopsy: comparable sperm retrieval rate in selected patients. Indian J Urol. 2012;28(1):37-42.

4. Westlander G, Ekerhovd E, Granberg S, Lycke N, Nilsson L, Werner C, Bergh C. Serial ultrasonography, hormonal profile and antisperm antibody response after testicular sperm aspiration. Hum Reprod. 2001;16(12):2621-7.

5. Harrington TG, Schauer D, Gilbert BR. Percutaneous testis biopsy: an alternative to open testicular biopsy in the evaluation of the subfertile man. J Urol. 1996:156(5):1647-51.

6. Sung HH, Ahn JS, Kim JJ, Choo SH, Han DH, Lee SW. The role of intracavernosal injection therapy and the reasons of withdrawal from therapy in patients with erectile dysfunction in the era of PDE5 inhibitors. Andrology. 2014:2(1):45-50.

7. Sharma R. Novel phosphodiesterase-5 inhibitors: current indications and future directions. Indian J Med Sci. 2007:61(12):667-79.

8. Yuan J, Zhang R, Yang Z, Lee J, Liu Y, Tian J, Qin X, Ren Z, Ding H, Chen Q, Mao C, Tang J. Comparative effectiveness and safety of oral phosphodiesterase type 5 inhibitors for erectile dysfunction: a systematic review and network meta-analysis. Eur Urol. 2013;63(5):902-12.

9. Stefanovic-Budimkic M, Jovanovic DR, Beslac-Bumbasirevic L, Ercegovac MD. Recurrent ischemic stroke associated with sildenafil and tadalafil use in a young adult. Clin Neurol Neurosurg. 2012;114(4):405-7.

Submit your next manuscript to BioMed Central and we will help you at every step:

- We accept pre-submission inquiries

- Our selector tool helps you to find the most relevant journal

- We provide round the clock customer support

- Convenient online submission

- Thorough peer review

- Inclusion in PubMed and all major indexing services

- Maximum visibility for your research 